\title{
Diferencias en el proceso de innovación en empresas pequeñas y medianas de la industria manufacturera de la ciudad de Chihuahua, México
}

Juan Óscar Ollivier

Fierro*

$\dagger$ Patricia Irene

Thompson Gutiérrez**

*Profesor de la Universidad Autónoma de Chihuahua Correo electrónico: jollivier@ uach.mx

**Profesora de la Universidad Autónoma de Chihuahua. Correo electrónico: jollivier@ uach.mx

${ }^{1}$ El acrónimo Pyme (Pequeñas y medianas empresas) ya incluye el plural.

\section{Resumen}

El objetivo de este trabajo es realizar un análisis comparativo de las principales variables que intervienen en el proceso de innovación entre las empresas pequeñas y medianas (Pyme $\left.{ }^{1}\right)$. El método se basó en una encuesta a 87 empresas Pyme manufactureras de la ciudad de Chihuahua, México, en 2007. Los principales resultados muestran una relación negativa entre la dinámica innovadora y el tamaño de la empresa, lo cual permite probar la hipótesis que postula una mayor eficiencia del proceso de innovación en las pequeñas empresas. Asimismo, se observa, en congruencia con la literatura, una relación positiva entre la dinámica innovadora y el desempeño económico relativo de las empresas, donde los clientes son la principal fuente de innovación. Se observan como áreas de oportunidad el desconocimiento general de los estímulos fiscales existentes, la escasa cultura del registro de la propiedad intelectual y la falta de articulación de las Pyme con los centros de investigación y las instituciones educativas. 
Palabras clave: Pyme, proceso de innovación, valor agregado, esfuerzo en I+D.

\title{
Differences in the innovation process between small and medium enterpri- ses in the manufacturing industry of Chihuahua, Mexico
}

\begin{abstract}
We undertake a comparative analysis of the main variables involved in the innovation process between small and medium size enterprises (SME). The method is based on a survey of eighty seven SME in the manufacturing industry of Chihuahua, Mexico in 2007. The main results show a negative relationship between the innovative dynamics and the size of the firms, which allows validating the hypothesis that postulates a higher efficiency in the innovative process of the smaller firms. It was also found, according to literature on the subject, a positive relationship between the innovation effort and the relative economic performance on the firms and also that customers are the main innovation source. The following are observed as opportunity areas: general ignorance of fiscal incentives; poor culture of intellectual property and lack of links between the SME and research \& development centers and educative institutions.
\end{abstract}

Key words: SME, Innovation process, Added value, R\&D effort.

\section{Introducción}

La cada vez mayor interdependencia de las economías nacionales, estimulada por el vertiginoso avance de la tecnología, particularmente en información y comunicaciones, ha incidido en la creación de un entorno más competitivo caracterizado por una demanda creciente de innovaciones, que ha provocado una reducción del ciclo de vida de los productos y de sus tecnologías.

Durante la mayor parte del siglo pasado, se consideró a la innovación, tanto de productos como de procesos, una actividad casi exclusiva de las grandes empresas, fruto de los esfuerzos en materia de investigación y desarrollo tecnológico (I+D) (Schumpeter, 1934 y Galbraith 1956). Sin embargo, en la actualidad, bajo el nuevo contexto económico, los estudios empíricos han demostrado que las pequeñas y medianas empresas han hecho contribuciones importantes al proceso de innovación general (Rothwell y Zegveld, 1982; Pavitt et al., 1987). A diferencia de las grandes empresas que cuentan con departamentos específicos de $\mathrm{I}+\mathrm{D}$, en las Pyme a partir del proceso creativo en el que emergen ideas potencialmente útiles a la 
empresa, éstas se van acumulando y articulando para dar lugar a la innovación que se concreta en un bien o servicio, que en el caso de los productos se ofrecen en el mercado (Pedroza y Sánchez, 2005).

$\mathrm{Al}$ encontrarse las Pyme cada vez más involucradas en los mercados competitivos globales, ya sea debido a su incorporación a las cadenas de suministro o a su expansión (Cagliano et al., 2001), la necesidad de innovar crece con el tiempo y se concentra en las ramas productivas más competidas y globalizadas, en las cuales se encuentran en desventaja las empresas de los países en desarrollo en general y particularmente su estrato más vulnerable que son las empresas pequeñas.

A pesar de que en las últimas décadas se han publicado numerosos estudios sobre la importancia del proceso de innovación en las pequeñas empresas manufactureras de los países industrializados (Rothwell, 1978, Smallbone, et al. 2003, McAdam, 2004, Carol y Mavis, 2007), las publicaciones sobre las características de este proceso en las Pyme manufactureras en los países en desarrollo son escasas y en Chihuahua prácticamente inexistentes. Adicionalmente, la mayor parte de estos estudios no distinguen entre las empresas pequeñas y medianas; se suele considerar en un solo grupo a las Pyme, cuando en realidad el espectro es amplio en los tamaños de las empresas que lo componen, que puede ir de uno a 250 trabajadores $\mathrm{y}$, por lo mismo, de un comportamiento que puede ser completamente diverso.

Dado lo anterior, el objetivo general de la investigación fue conocer más específicamente el proceso de innovación en las empresas pequeñas y medianas manufactureras de la ciudad de Chihuahua, particularmente la influencia que puede tener la innovación sobre los resultados económicos, distinguiendo entre los dos tamaños de empresas, para lograr una mejor comprensión del fenómeno y de esta forma avizorar soluciones a su problemática de acuerdo con su tamaño.

Los objetivos específicos que se desprenden de este objetivo general fueron: 1) identificar y analizar las principales variables que intervienen en el proceso de innovación, distinguiendo entre los estratos de pequeñas y medianas; y 2) analizar la relación entre el esfuerzo que realiza la empresa en innovación y su desempeño económico, para ello se compararon las empresas pequeñas con las medianas.

Los principales beneficiarios o usuarios potenciales de estos conocimientos son, en primer lugar, el medio académico donde se encuentran los investigadores y alumnos de posgrado que intervinieron en el trabajo; en segundo término, las ins- 
tituciones gubernamentales que requieren este tipo de información para el diseño e instrumentación de sus programas y, en tercer lugar, el sector empresarial de las Pyme para tomar conciencia de su realidad y de la importancia de las innovaciones en los resultados económicos.

En cuanto al concepto de innovación, se consideró la acepción del Manual de Oslo de la OCDE (1997), el cual señala que en la mayoría de los casos se percibe como una innovación tecnológica:

Las innovaciones tecnológicas de productos y procesos incluyen la implementación tecnológica de nuevos productos y procesos y mejoras tecnológicas significativas en productos y procesos. Una innovación ha sido implementada si ya ha sido introducida al mercado (innovación de producto) o usada dentro de un proceso de producción (innovación de proceso). ${ }^{2}$

Igualmente, se consideró importante el concepto de innovación definido por los investigadores mexicanos Corona y Jasso (2005), refiriéndose a que las empresas abarcan tanto novedades como adaptaciones, simples y complejas, de productos o ideas que se dirigen a un nuevo mercado.

La hipótesis planteada, relacionada con las empresas pequeñas y medianas de la industria manufacturera de la ciudad de Chihuahua, es: la relación entre el esfuerzo en $I+D$ y el desempeño económico es más eficiente en las empresas pequeñas que en las medianas.

\section{Método}

Se trata de un estudio empírico con un enfoque predominantemente cuantitativo, no experimental, transversal, de tipo descriptivo y correlacional, basado en una encuesta realizada en los años 2006 y 2007. Los sujetos de estudio fueron las empresas pequeñas y medianas de la industria manufacturera de la ciudad de Chihuahua, Chih., México, cuya población era del orden de 617, de acuerdo con un directorio construido por la Universidad Autónoma de Chihuahua (UACh), basado en la información proporcionada por la Cámara de la Industria de la Transformación (CANACINTRA) local, el Sistema de Información Empresarial Mexicano (SIEM) y

\footnotetext{
${ }^{2}$ Traducción del autor.
} 
la Asociación de Maquiladoras de Exportación, A.C. (AMEAC), el cual fue el marco muestral de donde se tomó la muestra.

Para el cálculo del tamaño de esta muestra se consideró una proporción de empresas que han realizado alguna innovación de un 50\%, con un $10 \%$ de error y un $95 \%$ de confianza (empleando la fórmula $n=p q Z^{2} / E^{2}$ ), resultando un tamaño calculado $n$ de 85 empresas. La muestra real fue de 87 empresas aleatoriamente seleccionadas del directorio de la UACh, de las cuales se encontró que 72 (83\%) habían realizado alguna innovación en el producto y/o en el proceso. Este resultado se puede comparar con el del trabajo de Carol y Mavis (2007) en una muestra de 877 Pyme en Taiwán, donde se encontró que el $80 \%$ de empresas realizó alguna innovación.

En el cuadro 1 se presentan las 72 empresas que realizaron alguna innovación según su subsector, distinguiendo los dos estratos analizados: las empresas pequeñas, de 1 a 50 trabajadores (que incluye las micro ${ }^{3}$ ); y las medianas, de 51 a 250 trabajadores.

Para la clasificación de las empresas del sector industrial de la industria manufacturera y sus subsectores, se consideró la Clasificación Mexicana de Actividades y Productos (CMAP), publicada por el Instituto Nacional de Estadística Geografía e Informática (INEGI, 2005): 31, Productos alimenticios, bebidas y tabaco (alimentos); 32, Textiles, prendas de vestir, e industria del cuero (textiles); 33, Industria de la madera incluyendo muebles (madera); 34, Papel y productos de papel, imprenta y editoriales (papel); 35, Sustancias químicas, productos derivados del petróleo y carbón, de hule y plástico (químicos); 36, Productos minerales no metálicos (minerales) y 38 , Productos metálicos, maquinaria y equipo, instrumentos quirúrgicos y de precisión (metálicos).

El instrumento ${ }^{4}$ utilizado fue un cuestionario que se contestó en entrevistas directas con los responsables de las empresas; cabe aclarar que dichas entrevistas fueron aplicadas por maestros y estudiantes de posgrado de la UACh.

\footnotetext{
${ }^{3}$ De acuerdo con la clasificación oficial, las empresas pequeñas incluyen a las empresas micro, lo cual es congruente con la clasificación internacional; sin embargo, en ocasiones para hacer énfasis en el grupo más numeroso, que es el de las empresas más pequeñas, se ha creado por razones prácticas la subcategoría de micro.

${ }^{4} \mathrm{El}$ autor pone a disposición del lector interesado el instrumento empleado en la encuesta.
} 


\section{Cuadro 1}

Empresas Pyme manufactureras según su subsector

\begin{tabular}{|c|cccccccc|}
\hline $\begin{array}{c}\text { Tamaño de } \\
\text { Empresas }\end{array}$ & Alimentos & Papel & Madera & Metálicos & Minerales & Químicos & Textil & Suma \\
\hline Pequeñas & 26 & 2 & 2 & 8 & 3 & 5 & 5 & 51 \\
Medianas & 9 & 2 & 1 & 3 & 0 & 4 & 2 & 21 \\
\hline Suma & 35 & 4 & 3 & 11 & 3 & 9 & 7 & 72 \\
\hline
\end{tabular}

Para fines de análisis, se consideraron los siete subsectores de la industria manufacturera en un solo grupo de empresas, distinguiéndolas en cuanto a su tamaño —dado el objetivo del trabajo- de acuerdo con la clasificación oficial en pequeñas y medianas. Sin embargo, cabe mencionar que el proceso de innovación puede tener aspectos comunes en la industria, pero también específicos en cada uno de los diferentes subsectores, tal como lo muestran los estudios realizados en Europa por Malerba et al. (2007) sobre el proceso de innovación en las empresas (de todos tamaños), en los diferentes sectores, como son el farmacéutico, biotecnología, equipo de telecomunicaciones, máquinas herramientas, software y de servicios.

\section{Resultados}

\section{Principales características de las empresas que han realizado innovaciones}

A continuación se muestran los principales resultados encontrados en las 72 empresas de la muestra que han realizado alguna innovación.

\section{a) Cantidad y escolaridad de los trabajadores}

En general, se encontró que la media del número de trabajadores es de 43.6 en las empresas de la muestra, correspondiendo a 10.9 en las pequeñas y a 123 en las medianas. Con respecto a los años de estudio de los trabajadores, considerando los ciclos de estudio terminados, se encontró una escolaridad media de 10.6 años, siendo ligeramente mayor en las empresas pequeñas (10.8), que en las medianas (10.2). Una posible explicación de este resultado es que las empresas pequeñas, dentro del espectro intensidad en mano de obra y capital, se ubican en un punto en el que son menos intensivas en mano de obra que las medianas.

Este resultado, en el que la escolaridad de los trabajadores es mayor en las empresas pequeñas que en las medianas, es congruente con lo encontrado en otras in- 
vestigaciones sobre el sector manufacturero de esta misma ciudad, que consideran todos los estratos de tamaños (Ollivier, 2005).

b) Realización de innovaciones al producto y al proceso

Se encontró que los productos que tienen alguna innovación (que puede ser en su proceso de producción) representan un $62.7 \%$ de las ventas de las empresas de la muestra, correspondiendo un $71 \%$ de las ventas de las pequeñas y un $37 \%$ de las medianas. Este resultado refleja una mayor importancia, interés y dependencia de la innovación en la economía de las empresas pequeñas que en las medianas.

En cuanto a las exportaciones, se encontró que el $6.5 \%$ del total de las ventas de productos que tienen alguna innovación se exporta, correspondiendo al $7.1 \%$ en el caso de las pequeñas y el 3.3\% de las medianas; sin embargo, en términos absolutos son mayores las exportaciones de las medianas. El hecho de que la proporción de las ventas de productos innovados, tanto nacionales como de exportación, sea mayor en las pequeñas es congruente con la literatura sobre el tema donde se muestra que las innovaciones son uno de los mayores determinantes en el desempeño exportador de las Pyme (Beise-Zee y C. Rammer, 2006). El cuadro 2 muestra la distribución de las empresas que han realizado estas innovaciones al producto $\mathrm{y} / \mathrm{o}$ al proceso de manufactura.

\section{Cuadro 2}

Número y porcentaje de empresas que han realizado alguna innovación al producto, al proceso, 0 a ambos según su tamaño

\begin{tabular}{|c|cccc|}
\hline $\begin{array}{c}\text { Tamaño de } \\
\text { empresas }\end{array}$ & $\begin{array}{c}\text { Innovación al } \\
\text { producto } \\
\text { (Número / \%) }\end{array}$ & $\begin{array}{c}\text { Innovación al } \\
\text { proceso } \\
\text { (Número / \%) }\end{array}$ & $\begin{array}{c}\text { Innovación a } \\
\text { ambos } \\
\text { (Número / \%) }\end{array}$ & $\begin{array}{c}\text { Suma } \\
\text { (Número / \%) }\end{array}$ \\
\hline Pequeñas & $18 / 35$ & $15 / 30$ & $18 / 35$ & $51 / 100$ \\
Medianas & $7 / 33$ & $8 / 38$ & $6 / 29$ & $21 / 100$ \\
\hline Suma & $25 / 35$ & $23 / 32$ & $24 / 33$ & $72 / 100$ \\
\hline
\end{tabular}

Aquí observamos que en las empresas pequeñas predominan las innovaciones a los productos sobre los procesos (35 vs. 30\%), mientras que en las medianas se tiene una mayor orientación hacia las innovaciones en los procesos (38 vs. 33\%). Además de una mayor intensidad innovadora en los productos de las empresas pequeñas, una posible explicación a este resultado se debe a que en las empresas medianas los procesos tienden a ser más complejos y, por lo mismo, con un margen mayor para la realización de innovaciones. 
Otra posible explicación es que en las empresas pequeñas los productos son menos maduros que en las medianas; además, dado que los productos (y sus tecnologías) avanzan en su ciclo de vida hacia la etapa de madurez las innovaciones tienden a trasladarse al proceso para incrementar su productividad y competitividad, como es el caso de la industria alimenticia (Castañón y Solleiro, 2007).

c) Dinamismo de la actividad de innovación

La característica de la dinámica innovadora se mide considerando dos variables: 1) la frecuencia de lanzamiento al mercado de productos que contengan alguna innovación, y 2) la duración del ciclo de innovación, que corresponde al periodo que transcurre desde que se concibe la idea hasta la comercialización (o producción en el caso de innovaciones en los procesos) del producto.

\section{Cuadro 3}

Frecuencia de lanzamiento al mercado de productos innovados y la duración del ciclo de innovación

\begin{tabular}{|c|cc|}
\hline Tamaño de empresas & $\begin{array}{c}\text { Frecuencia de lanzamiento de } \\
\text { un producto innovado } \\
\text { ( meses) }\end{array}$ & $\begin{array}{c}\text { Duración del ciclo de innovación } \\
\text { (meses) }\end{array}$ \\
\hline Pequeñas & 6.6 & 3.3 \\
Medianas & 15.1 & 9.4 \\
\hline Suma & 8.8 & 4.9 \\
\hline
\end{tabular}

En este cuadro observamos una mayor capacidad de respuesta al mercado en la medida en que las empresas son más pequeñas, lo cual se constata tanto en la frecuencia de lanzamiento de productos innovados al mercado, como en la duración del ciclo de innovación.

De manera similar, estos resultados son congruentes con los realizados en los Estados Unidos, en los que se ha encontrado a nivel macro una relación negativa entre el tamaño de la Pyme y su propensión a innovar (Kalantaridis y Pheby, 1999). Por su parte, estas variables que expresan la dinámica innovadora son comparables al indicador NPDP (del inglés New Product Development Process), el cual es considerado como una medición de las mejores prácticas para incrementar la competitividad y penetración en los mercados (O Shea, y Mc Bain, 1999). 
d) Motivación de las innovaciones

Con respecto a las principales motivaciones para la realización de las innovaciones se encontró en orden de importancia decreciente: 1) mejorar la calidad de los productos, 2) disminuir costos y precio, 3) disminuir tiempos de fabricación y 4) mejorar diseño de los productos; las primeras tres motivaciones están estrechamente relacionadas con las innovaciones en el proceso y la cuarta con el producto. Por otra parte, este resultado confirma que el deseo por mejorar la competitividad de los productos es un motor de las innovaciones.

e) Fuentes de ideas de las innovaciones.

En cuanto a las principales fuentes de ideas para las innovaciones se encontró lo siguiente en orden de importancia decreciente: 1) los clientes, 2) los proveedores, 3) Internet, 4) revistas y 5) libros. Lo anterior es congruente con el inciso anterior en el sentido de que la satisfacción del cliente, en la cual la calidad juega un papel de primera importancia, es uno de los principales impulsores de las innovaciones, mismo que corresponde a una relación con el cliente de tipo "jalar" (del inglés customer-pull approach), recomendada para el desarrollo de innovaciones en los productos (Temtime y Solomon, 2002; Gunasekaran et al., 1996).

Vale la pena aclarar que una gran parte de los clientes de las empresas Pyme de Chihuahua son maquiladoras (aproximadamente 70 en la ciudad y su zona de influencia), la mayor parte grandes trasnacionales que manejan estándares tecnológicos relativamente altos, que propician una transferencia de tecnología en el proceso de proveeduría de las Pyme a ellas, la cual puede ser vista como una externalidad positiva. Asimismo, esta transferencia de tecnología se realiza a través de la movilidad de los trabajadores de las empresas trasnacionales a las Pyme locales (Vera-Cruz y Dutrénit, 2007).

\section{f) Alianza con otra organización}

Se encontró que el $42 \%$ de la muestra tiene una alianza con otra organización para el desarrollo de las innovaciones, en orden decreciente sería de la siguiente manera: 1) otras empresas, principalmente proveedores y clientes; 2) centros de investigación; 3) instituciones de educación y 4) otras divisiones de la misma empresa. Este resultado es congruente con el anterior en el sentido de la importancia del papel que 
juegan los clientes en el desarrollo de las innovaciones. Asimismo, se observó en el análisis la escasa relación que tienen las Pyme con las instituciones de educación.

De igual forma, este resultado es congruente con lo encontrado por T. Allen et al. (1983) en pequeñas empresas en Irlanda, España y México, en el sentido de que la fuente principal de las ideas de innovación son las otras empresas, mientras que las universidades y centros de investigación son menos frecuentes.

Por otra parte, en cuanto a la adquisición de una licencia tecnológica a otra empresa, sólo el $5.5 \%$ de las empresas lo ha realizado, todas ellas pequeñas.

g) Registro de la propiedad intelectual de las innovaciones

\section{Cuadro 4}

Número y porcentaje de empresas que han hecho o están haciendo algún trámite de registro de propiedad intelectual según su tamaño

\begin{tabular}{|c|ccccc|}
\hline $\begin{array}{c}\text { Tamaño de } \\
\text { empresas }\end{array}$ & $\begin{array}{c}\text { Patente } \\
\text { (Número / \%) }\end{array}$ & $\begin{array}{c}\text { Marca } \\
\text { (Número / \%) }\end{array}$ & $\begin{array}{c}\text { Derechos de autor } \\
\text { (Número / \%) }\end{array}$ & $\begin{array}{c}\text { Otro* } \\
\text { (Número / \%) }\end{array}$ & $\begin{array}{c}\text { Suma } \\
\text { (Número / \%) }\end{array}$ \\
\hline Pequeñas & $6 / 12$ & $10 / 20$ & $1 / 2$ & $2 / 4$ & $19 / 38$ \\
Medianas & $4 / 19$ & $8 / 38$ & $0 / 0$ & $1 / 5$ & $13 / 62$ \\
\hline Suma & $10 / 14$ & $18 / 25$ & $1 / 1.4$ & $3 / 4.2$ & $32 / 44.6$ \\
\hline
\end{tabular}

* En el rubro de "Otro" se encuentran los modelos de utilidad, diseños y secretos industriales

En el cuadro anterior observamos que de las empresas que han realizado alguna innovación a sus productos o procesos, sólo el 14\% ha registrado o está registrando una patente, que corresponde a un $12 \%$ de las pequeñas y un $19 \%$ de las medianas, por lo que se observa una mayor cultura de protección intelectual en estas últimas empresas. Del mismo modo, vemos que el 38\% de las empresas pequeñas han realizado algún registro de propiedad intelectual, mientras que en las medianas el $62 \%$ lo ha efectuado.

h) Personal dedicado a la investigación y desarrollo tecnológico

Con relación al esfuerzo que realizan las empresas en I+D, medido por el número de trabajadores que laboran en estas actividades en cualesquiera de sus áreas, se encontró - en equivalentes de tiempo completo ETC (FTE en inglés de Full Time 
Equivalent) - a 1.56 personas para las empresas en general, que corresponden a 0.28 personas en el caso de las pequeñas y 4.8 en el de las medianas.

Si estas cifras las comparamos con el número de trabajadores de los dos estratos de la muestra se tiene que en las pequeñas corresponde a un $2.6 \%$ de los trabajadores y en las medianas a un $3.9 \%$ de los mismos. Este resultado, en el sentido de que es menor en términos relativos el personal que se dedica a la $\mathrm{I}+\mathrm{D}$ en las pequeñas que en las medianas empresas es congruente con lo encontrado por McAdam et al. (2004) en una encuesta realizada a 2,086 Pyme en Irlanda, en el sentido de que las pequeñas empresas están más enfocadas en la operación de la misma por estar más influidas por factores contingentes y accidentales.

\section{i) Inversión en investigación y desarrollo tecnológico}

Este mismo esfuerzo, medido por el porcentaje de la inversión en I+D sobre las ventas, fue de un $7.6 \%$ para las empresas en general, correspondiendo a un $6.8 \%$ para las pequeñas y un $9.7 \%$ para las medianas. Cabe mencionar que estos recursos son estimados con base a la distribución de las actividades de I+D en las diferentes áreas de la empresa, dado que en la gran mayoría de ellas (79\%) no cuenta con un departamento o área especial para realizar estas actividades.

Por lo que respecta al origen de estos recursos, el 37.5\% de las empresas ha solicitado un financiamiento para realizar actividades relacionadas a la I+D, sólo lo obtuvieron el $29.2 \%$ : el $4.7 \%$ del gobierno y $24.5 \%$ de la banca privada, cabe señalar que esta última es también parcialmente fondeada por la banca de desarrollo para los proyectos en I+D. El monto promedio de estos préstamos ha sido de 198,678 dls para las empresas en general, correspondiendo a 22,500 dls en el caso de las pequeñas y de 410,091 dls en el caso de las medianas.

\section{j) Uso del estímulo fiscal}

En cuanto al aprovechamiento del incentivo económico del gobierno federal mexicano, que permite considerar como un crédito fiscal para el pago del Impuesto Sobre la Renta (ISR) hasta el 30\% de las inversiones en investigación y desarrollo tecnológico $(\mathrm{I}+\mathrm{D})^{4}$, se encontró que sólo el $8.3 \%$ de las empresas de la muestra

\footnotetext{
${ }^{5}$ El artículo 219 de la Ley sobre el ISR, reformado el 30/12/2002 estipula este estímulo fiscal para las empresas en general.
} 
lo ha aprovechado, que corresponde a un $7.8 \%$ de las pequeñas y un $9.5 \%$ de las medianas, lo cual pone en evidencia el escaso conocimiento por parte de las Pyme, en general, y de las pequeñas, en particular, de este programa de apoyo a las actividades de I+D iniciado en el 2002.

k) Edad del equipamiento de las empresas

La edad promedio del equipo productivo en los dos estratos es de 11.7 años, de 10.1 para las pequeñas y de 18.33 para las medianas, lo cual muestra una relativa obsolescencia del equipamiento de las Pyme en general y de las medianas en particular.

1) Principales resultados económicos de las empresas de la muestra

\section{Cuadro 5}

Ventas anuales, valor agregado y utilidades en porcentaje de las ventas de las empresas según su tamaño

\begin{tabular}{|c|ccc|}
\hline Tamaño de empresas & $\begin{array}{c}\text { Ventas anuales } \\
(\mathrm{dls}, 2005)\end{array}$ & $\begin{array}{c}\text { Valor agregado } \\
(\%)\end{array}$ & $\begin{array}{c}\text { Utilidades antes de } \\
\text { impuestos }(\%)\end{array}$ \\
\hline Pequeñas & 500,958 & 45.8 & 24.8 \\
Medianas & $4,454,545$ & 42.6 & 20.2 \\
\hline Suma & $1,636,363$ & 44.9 & 23.5 \\
\hline
\end{tabular}

Es evidente que tanto el valor agregado como las utilidades son mayores en términos relativos en las pequeñas empresas que en las medianas. La relación positiva entre la innovación, tanto en productos como en procesos con el desempeño de negocios de las empresas Pyme, es confirmada por los hallazgos de investigaciones en diversos países como es el caso del Reino Unido (Smallbone et al., 2003).

\section{Análisis de regresión de las principales variables}

El análisis de correlaciones de las variables principales muestra una correlación positiva significativa entre el esfuerzo relativo en I+D (porcentaje de la inversión en I+D sobre las ventas) y el porcentaje del valor agregado, lo cual sugiere que a mayor esfuerzo en I+D se tiene un mayor valor agregado, cuyos dos grandes componentes son sueldos y utilidades. 
A partir de la relación anterior, se construye un modelo de regresión lineal simple teniendo como variable dependiente el valor agregado y como predictora el esfuerzo relativo en I+D, ambas en términos porcentuales de las ventas. Con el fin de observar la diferencia entre los dos tamaños de empresas, a continuación se muestran los valores de los dos modelos de regresión (ambos con un valor de $\mathrm{F}=$ 5.9) en los siguientes cuadros.

\section{Cuadro 6}

\section{Modelo de regresión de las empresas pequeñas}

\begin{tabular}{|c|ccccc|}
\hline Dependiente & Predictora & Coef. B & Coef. Beta & t & Sig. \\
\hline $\begin{array}{c}\text { Valor agregado (\% } \\
\text { de ventas) }\end{array}$ & $\begin{array}{c}\text { Inversión en I+D } \\
\text { (\% de ventas) }\end{array}$ & 0.645 & 0.357 & 2.45 & 0.019 \\
\hline & Constante & 41.5 & & 16.32 & 0.000 \\
\hline
\end{tabular}

\section{Cuadro 7}

Modelo de regresión de las empresas medianas

\begin{tabular}{|c|ccccc|}
\hline Dependiente & Predictora & Coef. b & Coef. Beta & $\mathrm{t}$ & Sig. \\
\hline $\begin{array}{c}\text { Valor agregado } \\
\text { (\% de ventas })\end{array}$ & $\begin{array}{c}\text { Inversión en I+D } \\
\text { (\% de ventas })\end{array}$ & 0.902 & 0.533 & 2.44 & 0.027 \\
\hline & Constante & 33.3 & & 6.69 & 0.000 \\
\hline
\end{tabular}

Figura 1

Modelos de regresión lineal entre valor agregado y esfuerzo en I+D (en porcentaje para las empresas pequeñas y medianas)

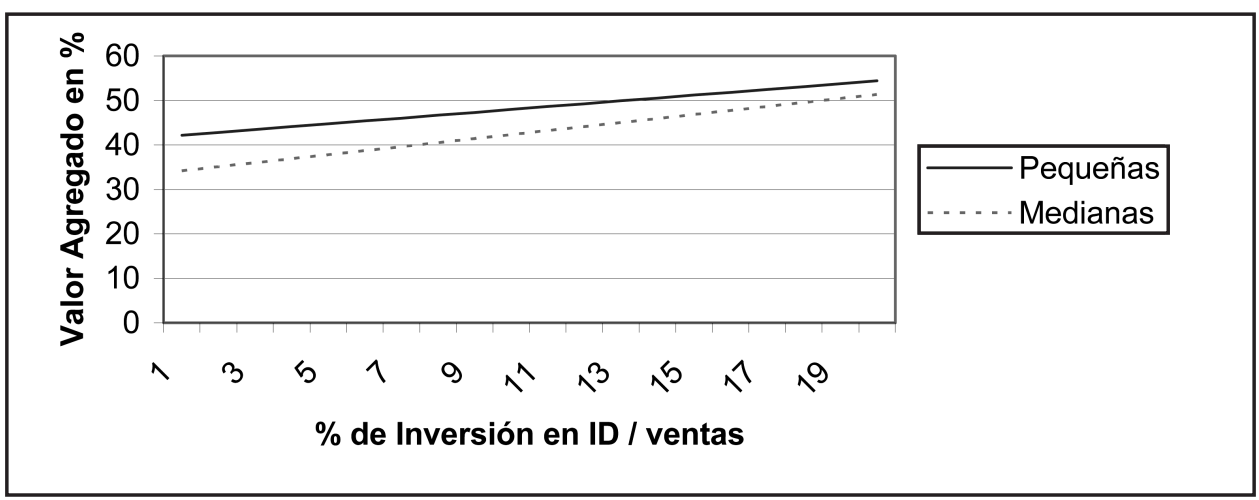


En esta gráfica se observa que para el mismo esfuerzo en I+D se tiene una mayor valor agregado en las empresas pequeñas que en las medianas; es decir que el efecto de la inversión en I+D sobre el valor agregado es inversamente proporcionales al tamaño de las empresas.

En relación con la diferencia, debido al tamaño de las empresas en el efecto de la I+D sobre los resultados económicos, es congruente con el estudio realizado por Cagliano et al. (2001) en una amplia muestra internacional de Pyme (igualmente hasta 250 trabajadores), en el que se encuentran efectos sustanciales en las prácticas de innovación por el tamaño de las empresas.

\section{Discusión}

Los resultados anteriores permiten visualizar algunos aspectos en los que existe una clara distinción entre las empresas pequeñas y medianas en cuanto al proceso de innovación. No obstante que los esfuerzos para la I+D - medidos por el personal dedicado, las inversiones y la protección intelectual - son mayores en las empresas medianas. Por otra parte, los resultados económicos relativos - medidos por las ventas de productos innovados, la exportación de los mismos, el valor agregado y la utilidad antes de impuestos - son mayores en las empresas pequeñas, lo cual coincide con lo encontrado por Acs y Audretsch (1988) en las Pyme de los EUA.

Congruente con lo anterior, el análisis de regresión lineal (ver figura 1) permite probar la hipótesis formulada: la relación entre el esfuerzo en $I+D$ y el desempeño económico es más eficiente en las empresas pequeñas que en las medianas. En este análisis se constata que la relación entre las variables valor agregado y esfuerzo de innovación es más eficiente en las pequeñas empresas que en las medianas; esto se observa a través del valor del coeficiente de la constante donde la intersección es mayor en las pequeñas (41.5) que en las medianas (33.3) en el rango considerado y que las líneas son prácticamente paralelas en un rango amplio.

Una posible explicación de estos resultados es el conjunto de aspectos en los que se observó destacan las pequeñas empresas, como son: 1) una ligera mayor escolaridad de los trabajadores; 2) un mayor énfasis en las innovaciones en el producto $v s$. el proceso; 3 ) un mayor dinamismo en el proceso de innovación, medido por la frecuencia de lanzamiento de innovaciones y la duración del ciclo de innovación; y 4) una menor edad en el equipo productivo. 
Adicionalmente se encuentra un factor subyacente que influye en todas las actividades de la empresa que se encuentra estrechamente relacionado al tamaño pequeño de la empresa; la flexibilidad de la misma, particularmente útil al permitir procesos de toma de decisiones más ágiles. De acuerdo con el estudio realizado por Wiele y Brown (1998) en las Pyme australianas, la principal razón a la que obedece la mayor agilidad en las empresas pequeñas es la rapidez con la que se toman las decisiones en estas empresas.

Otro resultado que vale la pena destacar, en ambos tamaños de empresas, es la relación con el cliente, como motor del proceso de innovación; es decir, la proximidad y conocimiento del cliente son factores claves en la innovación de las Pyme (Voss, 1998), que a su vez estos factores son la base de las economías por aglomeración realizadas por los agrupamientos industriales, mejor conocidos como clusters en inglés. Sin embargo, el hecho de pertenecer a un cluster no garantiza por sí mismo la innovación, sino que la dinámica innovadora de una empresa puede ser estimulada por las relaciones y el clima de trabajo generado por otras empresas innovadoras del agrupamiento industrial (Beaudry y Breschi, 2003).

\section{Conclusiones}

De los resultados y discusión anteriores, se pueden extraer las siguientes conclusiones para las empresas Pyme manufactureras de la ciudad de Chihuahua.

1. Las empresas medianas realizan un mayor esfuerzo de innovación en relación con las pequeñas tanto en términos absolutos como relativos en cuanto al personal dedicado y a las inversiones en I+D; los registros de protección intelectual de las innovaciones y el aprovechamiento del estímulo fiscal del ISR (incisos g, h, i, j y cuadro 4 de resultados).

2. Las empresas pequeñas tienen una dinámica innovadora más intensa en relación con las medianas, observada a través de una mayor frecuencia de lanzamiento de productos innovados; una menor duración en el ciclo del proceso de innovación; la proporción de productos innovados en las ventas nacionales y de exportación (inciso b y cuadro 3 de resultados).

3. Por otra parte, las empresas pequeñas tienen un mejor desempeño económico relativo, expresado por mayores porcentajes sobre las ventas del valor agregado y la utilidad antes de impuestos, lo cual se sugiere es influido por la mayor dinámica innovadora señalada en el punto precedente (cuadro 5). 
4. Congruente con lo anterior, el análisis de regresión lineal permite probar la hipótesis formulada: la relación entre el esfuerzo en $I+D$ y el desempeño económico es más eficiente en las empresas pequeñas que en las medianas (la evidencia estadística se muestra en los cuadros 6 y 7 y figura 1 y se analiza en la discusión). En este análisis se constata que la relación entre las variables valor agregado y esfuerzo de innovación es más eficiente en las pequeñas empresas que en las medianas. Como explicación de esta hipótesis, adicionalmente a la mayor dinámica innovadora, se encontró una ligera mayor escolaridad de los trabajadores; un mayor énfasis en las innovaciones en el producto vs. el proceso; una menor edad en el equipo productivo y la flexibilidad propia de las empresas pequeñas (incisos a, $\mathrm{k}$ y cuadro 2 de resultados).

5. La relación con el cliente es común a ambos estratos como motor del proceso de innovación, donde la proximidad y conocimiento del cliente son factores clave en la innovación de las Pyme, que a su vez son la base de las economías por aglomeración realizadas por los agrupamientos (clusters en inglés), industriales (incisos d y e de resultados).

Como conclusión general, este trabajo aporta indicios sobre el complejo fenómeno del proceso de innovación en las empresas pequeñas y medianas manufactureras, lo cual permite una mejor comprensión del mismo visualizando las diferencias entre los dos tamaños de empresas, localizadas en este caso en una ciudad del norte de México. Finalmente, se considera que la innovación es un factor cada vez más importante en la competitividad de las empresas, las cuales en su conjunto determinan la competitividad de los países.

\section{Recomendaciones}

Con relación a las áreas de oportunidad identificadas y sus posibles opciones de solución se recomiendan investigaciones de una mayor profundidad para la adecuada instrumentación de las siguientes medidas:

- Una mayor difusión del estímulo del crédito fiscal sobre el ISR, para las actividades de I+D que establece el artículo 219 de la LISR.

- El fortalecimiento de la cultura de registrar la propiedad intelectual de sus innovaciones, por medio de patentes, marcas, modelos, diseño y secretos industriales. 
- Un reforzamiento de la vinculación con las instituciones educativas y centros de investigación, para la realización de proyectos conjuntos de I+D.

Sobre este último punto se identificó que parte de los principales problemas que obstaculizan el proceso de innovación tienen como denominador común la falta de vinculación entre las empresas con los sectores educativos y centros de investigación. Una de las soluciones que se ha adoptado en otros países, con diferente éxito en la solución de la problemática anterior, ha sido procurar la sinergia entre los actores a través de la creación de los sistemas (regionales o nacionales) de innovación (SRI O SNI), cuya función sustantiva es la de crear el espacio de comunicación y generación de vínculos entre los tres agentes clave del proceso innovador: el sector productivo, el educativo y el gubernamental.

Tal como lo recomienda el especialista regional en planificación y gestión de ciencia y tecnología de la UNESCO, Eduardo Martínez (2005), refiriéndose a un SNI en América Latina:

[...] este sistema implicaría el desarrollo de una red de instituciones, de recursos, de interacciones y relaciones, de mecanismo e instrumentos de política y de actividades científicas y tecnológicas, que promuevan, articulen y materialicen los procesos de innovación y difusión tecnológica en la sociedad.

Finalmente, tal como anteriormente se mencionó, debido a las diferencias en el proceso de innovación entre los subsectores de la industria manufacturera se recomienda realizar el mismo estudio seleccionando una muestra estratificada de estos subsectores para poder analizarlos en forma individual, así como compararlos entre ellos desde el punto de vista del proceso de innovación.

\section{Bibliografía}

Acs, Z y D. AudReTSCH (1988), "Innovation and Firm size in Manufacturing", Technovation, Vol 7, No. 3, pp 197-211.

Allen, T., D. Hayman y D. Pinckeney (1983), "Transferring Technology to the Small manufacturing Firm: A Study of Technology Transfer in Three Countries", Research Policy, Vol. 12, No. 4, pp. 199-212.

BeAudry, C. y S. BReschi (2003), "Are firms in clusters really more innovative ?", Economics of Innovation and New Technology, Issue 4, agosto, pp. 325-342. 
Beise-Zee, R. y C. Rammer (2006), "Local User-Producer Interaction in Innovation and Export Performance of Firms", Small Business Economics, No. 27, pp. 207-222.

Cagliano, R., K. Blackmon y C. Voss (2001), "Small firms Ander the MiCROSCOPE: Internacional differences in production/operations management practices and performance", Integrated Manufacturing Systems, Vol. 12, pp. 469-482.

Carol Yeh-Yun Lin y Mavis Yi-Ching Chen (2007), "Does innovation lead to performance? An empirical study of SME in Taiwan", Management Research News, Vol. 30, No. 2, p. 115.

CAstañón, R y J. L. Solleiro (2007), "Los instrumentos de política industrial y la competitividad de las pequeñas y medianas empresas mexicanas del sector de alimentos", en Innovación y desarrollo tecnológico, Universidad de Guadalajara.

Corona, L. y J. JASso, (2005), "Enfoques y características de la sociedad del conocimiento. Evolución y perspectivas para México", Innovación en la sociedad del conocimiento, Puebla, Benemérita Universidad Autónoma de Puebla, México.

Galbraith, J. K. (1956), The affluent Society, Boston, Houghton Mifflin.

Gunasekaran, A., P. Окко, T. Martikainen y P. Yli-Olli (1996), "Improving productivity and Quality in small and medium enterprises: Cases and analysis", International Small Business Journal, Vol. 15, pp. 59-72.

Instituto Nacional de Estadística Geografía e Informática (2005), Censo Económico 2004, industria manufacturera, México: INEGI.

Kalantaridis, C. y J. Pheby (1999), "Processes of innovation among manufacturing SMEs: The experience of Bedfordshire", Entrepreneurship \& Regional Development, Vol. 11, Iss. 1, pp. 57-78.

Malerba, F., R. Nelson, L. Orsenigo y S. Winter (2007), "Demand, innovation and the dynamics of market Structure: The role of experimental users and diverse preferences", Journal of Evolutionary Economics, Springer, Vol 17(4), agosto, pp 371399.

Martínez, E. (2005), "Ciencia, tecnología y Estado en América Latina: nuevo siglo y nuevo paradigma”, Innovación en la sociedad del conocimiento, Puebla, Benemérita Universidad Autónoma de Puebla, México. 
McAdam, R., R. Reid y D. Gibson (2004), "Innovation and organizational size in Irish SME: an empirical study", International Journal of Innovation Management, Vol. 8, No 2, pp. 147-165.

Nonaka, I. y K. Takeuchi (1995), The Knowledge Creating Company: How Japanese Companies Create the Dynamics of Innovation Oxford: Oxford University Press.

OECD (1997), Propoved Guidelines for Collecting and Interpreting Technological Innovation Data. Oslo Manual, Paris: OECD

Ollivier, J. (2005), "El problema del rezago tecnológico de las microempresas industriales y alternativas de solución", en Contaduría y Administración, FCA, UNAM, Vol. 221, pp. 137-160.

O Shea, A. y N. Mcbain (1999), "The process of innovation in small manufacturing firms", International Journal of Technology Management, Vol. 18, Núms. 5,6,7,8, pp. 610-627.

PavitT, K., M. Robson y J. Towsend (1987), "The size of the innovating firms in the UK: 1945-1983”, The Journal of Industrial Economics, Vol. 55, pp. 297-316.

Pedroza, A. y J. SÁnchez (2005), Procesos de innovación tecnológica en la pequeña y mediana empresa, Guadalajara, Universidad de Guadalajara.

Rothwell, R. (1978) "Small and Medium sized Manufacturing Firms and Technological Innovation”, Management Decision, Vol. 16, No. 6, London p. 362.

y W. Zegveld (1982), Innovation and Small and Medium Sized Enterprises, London, Pinter.

Schumpeter, J. (1934), The Theory of Economic Development, Cambridge, MA, Cambridge University Press.

Smallbone, D., D. North, S. Ropere e I. Vickers (2003), "Innovation and the use of technology in manufacturing plants and SMEs: an interregional comparison", Environment \& Planning C: Government \& Policy, Vol. 21, Iss. 1, pp. 37-53.

Temtime, Z y G. Solomon (2002), "Total quality management and the planning behavior of SMEs in developing economies", The TQM Magazine, Vol. 14, pp. 181-191. 
Vera-Cruz, A y G. Dutrénit (2007), "Las derramas de las MNC a través de la movilidad de los trabajadores", en Innovación y desarrollo tecnológico, Universidad de Guadalajara.

Voss, C. (1998), "Made in Europe: Small companies", Business Strategy Review, Vol. 9, pp. 1-19.

Wiele, T y A. BRown (1998), "Venturing down the TQM path for SME's", International Small Business Journal, Vol. 16, pp. 50-69.

(A) 